\title{
Genetic inactivation of alpha-synuclein affects embryonic development of dopaminergic neurons of the substantia nigra, but not the ventral tegmental area, in mouse brain
}

Tatyana V. Tarasova ${ }^{1}$, Olga A. Lytkina $^{1}{ }^{\text {, }}$ Valeria V. Goloborshcheva ${ }^{1}$, Larisa N. Skuratovskaya ${ }^{2}$, Alexandr I. Antohin ${ }^{3}$, Ruslan K. Ovchinnikov ${ }^{1,3}$, Michail S. Kukharsky ${ }^{\text {Corresp. } 1}$

1 Laboratory of Genetic Modeling of Neurodegenerative Processes, Institute of Physiologically Active Compounds, Russian Academy of Sciences, Chernogolovka, Russia

2 Institute of general pathology and pathophysiology, Moscow, Russia

3 Faculty of Biomedical Science, Pirogov Russian National Research Medical University, Moscow, Russia

Corresponding Author: Michail S. Kukharsky

Email address: kukharskym@ipac.ac.ru

Lesion of the dopaminergic neurons of the nigrostriatal system is a key feature of Parkinson's disease (PD). Alpha-synuclein is a protein that is a major component of Lewy bodies, histopathological hallmarks of PD, and is involved in regulation of dopamine (DA) neurotransmission. Previous studies of knockout mice have shown that inactivation of alpha-synuclein gene can lead to the reduction in number of DA neurons in the substantia nigra (SN). DA neurons of the SN are known to be the most affected in PD patients whereas DA neurons of neighboring ventral tegmental area (VTA) are much less susceptible to degeneration. Here we have studied the dynamics of changes in TH-positive cell numbers in the SN and VTA during a critical period of their embryonic development in alpha-synuclein knockout mice. This precise study of DA neurons during development of the SN revealed that not only is the number of DA neurons reduced by the end of the period of ontogenic selection, but that the way these neurons are formed is altered in alpha-synuclein knockout mice. At the same time, DA neurons in the VTA are not affected. Alpha-synuclein exerts a modulating effect on the formation of DA neurons in the SN and has no effect on the formation of DA neurons in VTA, the structure that is much less susceptible to degeneration in PD brain, suggesting a potential role of alpha-synuclein in the development of the population of DA neurons in substantia nigra. 
1 Genetic inactivation of alpha-synuclein affects embryonic development of 2 dopaminergic neurons of the substantia nigra, but not the ventral tegmental 3 area in mouse brain

4

5 Tatiana V. Tarasova ${ }^{1}$, Olga A. Lytkina ${ }^{1}$, Valeria V. Goloborshcheva ${ }^{1}$, Larisa N. 6 Skuratovskaya ${ }^{2}$, Alexandr I. Antohin ${ }^{3}$, Ruslan K. Ovchinnikov ${ }^{1,3}$, Michail S. 7 Kukharsky ${ }^{1, *}$

$9{ }^{1}$ Laboratory of Genetic Modeling of Neurodegenerative Processes, Institute of 10 Physiologically Active Compounds, Russian Academy of Sciences, Chernogolovka, 11 Moscow region, Russia

12 Institute of general pathology and pathophysiology, Moscow, Russia

13 3 Faculty of Biomedical Science, Pirogov Russian National Research Medical 14 University, Moscow, Russia

* Corresponding Author:

18 Email address: kukharskym@ipac.ac.ru 


\section{Abstract}

Lesion of the dopaminergic neurons of the nigrostriatal system is a key feature of Parkinson's disease (PD). Alpha-synuclein is a protein that is a major component of Lewy bodies, histopathological hallmarks of PD, and is involved in regulation of dopamine (DA) neurotransmission. Previous studies of knockout mice have shown that inactivation of alpha-synuclein gene can lead to the reduction in number of DA neurons in the substantia nigra (SN). DA neurons of the $\mathrm{SN}$ are known to be the most affected in PD patients whereas DA neurons of neighboring ventral tegmental area (VTA) are much less susceptible to degeneration. Here we have studied the dynamics of changes in TH-positive cell numbers in the SN and VTA during a critical period of their embryonic development in alpha-synuclein knockout mice. This precise study of DA neurons during development of the SN revealed that not only is the number of DA neurons reduced by the end of the period of ontogenic selection, but that the way these neurons are formed is altered in alpha-synuclein knockout mice. At the same time, DA neurons in the VTA are not affected. Alphasynuclein exerts a modulating effect on the formation of DA neurons in the SN and has no effect on the formation of DA neurons in VTA, the structure that is much less susceptible to degeneration in PD brain, suggesting a potential role of alphasynuclein in the development of the population of DA neurons in substantia nigra.

\section{Introduction}

Alpha-synuclein, a small natively unfolded protein abundantly expressed in vertebrate neurons, is involved in pathogenesis of certain neurodegenerative diseases. Principal members of this group of diseases known as alphasynucleinopathies include, Parkinson's disease, Lewy body dementia and multisystem atrophy, but alpha-synuclein pathology is often observed in the nervous system of patients with other neurodegenerative diseases (Goedert et al. 2017).

Although the normal functions of alpha-synuclein are still not fully understood, there is a growing body of evidence that this protein modulates the efficiency of various mechanisms that are important for neuronal physiology, including synaptic vesicle function and synaptic transmission (Burre et al. 2010; Greten-Harrison et al. 2010; Janezic et al. 2013; Nemani et al. 2010; Ninkina et al. 2012; Vargas et al. 2017), mitochondrial ATP production (Ludtmann et al. 2016; Ryan et al. 2015), survival (Gorbatyuk et al. 2010; Robertson et al. 2004), and sensitivity to neurotoxins [9]. The key mechanism of pathology of PD, severe loss of DA neurons of the SN, remains a major focus of research on the way to revealing specific molecular targets for developing the disease-modifying drugs. The main findings describing the various factors that are involved in the development and maturation of DA neurons are summarized by Luo \& Huang (Luo \& Huang 2016). It is believed that the period between mouse embryonic days 11.5 and 13.5 when post-mitotic precursors of DA 
60 neurons are migrating from the ventricular zone located on the border between

61 midbrain and forebrain to their sites in the SN and VTA where their final

62 differentiation takes place, is a critical period in the process of forming these two

63 neuronal populations. This coincides with a dramatic increase of alpha-synuclein

64 expression in midbrain nuclei (Abeliovich \& Hammond 2007). Moreover, depletion

65 of alpha-synuclein from developing neurons, leads to a smaller number of mature

66 DA neurons in the SN of knockout mice (Garcia-Reitboeck et al. 2013). The aim of

67 this study was to reveal whether alpha-synuclein could have a different impact on

68 the development of DA neurons in the VTA that are much less affected by in two close populations, the SN and VTA, were analyzed during embryonic development of alpha-synuclein knockout mice.

\section{Materials and Methods}

All animal work was carried out in accordance with the Rules of Good Laboratory Practice in Russian Federation (2016). Bioethics committee of Institute of Physiologically Active Compounds, Russian Academy of Sciences provided full approval for this research (Approval №20 dated 23.06.2017). A line of alphasynuclein knockout mice on C57B16J background that was used in this study has been described previously (Abeliovich et al. 2000; Robertson et al. 2004). The embryos were fixed in cold 4\% paraformaldehyde solution, paraffin-embedded, and serial $8 \mu \mathrm{m}$ transverse sections through the mesencephalon regions were prepared for immunostaining as described previously (Robertson et al. 2004). Dopaminergic neurons were visualised by staining sections with anti-tyrosine hydroxylase (TH) antibodies (mouse monoclonal antibody, clone TH-2, Sigma) diluted 1:1000. Stereological counting of TH-positive cells through the entire lateral region of the mesencephalon was performed as described previously (Al-Wandi et al. 2010; Robertson et al. 2004). Statistical analysis was performed using GraphPad Prism software (GraphPad, San Diego, CA, USA). A probability value of $\mathrm{p}<0.05$ was considered statistically significant.

\section{Results}

In this study, we analysed the sub-population of developing dopaminergic neurons within the substantia nigra in alpha-synuclein knockout mice (aKO) and control wild type (WT) littermates on embryonic days E10.5, E11.5, E12.5 and E13.5. The total numbers of TH-positive cells were estimated in the whole SN regions at each embryonic stage. A small number of TH-positive cells in the lateral region of the mesencephalon that represent the primordium of the $\mathrm{SN}$ were already detected by embryonic day E10.5 and no difference between knock-out and WT mice were revealed (Fig.1A and Supplementary material). In WT mice no substantial increase 
100 in the number of these neurons were observed during the next embryonic day, while

101 in aKO mice, a nearly four-fold increase was detected. As a result, the number of

102 TH-positive cells was 2.9 times higher in the SN of E11.5 aKO embryos than in WT

103 embryos at the same stage (Fig.1A, Fig.2). A sharp increase in number of TH-

104 positive neurons was observed between E11.5 and E12.5 in WT mice while in aKO

105 mice this increase was less prominent. Whereas aKO mice show an increased

106 number of neurons one day earlier (E11.5), they eventually show a comparatively

107 lower number of TH-positive neurons at all of the following days where

108 measurements were obtained. Therefore, at E12.5, more SN neurons were found in

109 WT than in aKO embryos. At this point the number of TH-positive neurons have

110 reached their maximum number followed by a decline in number on day E13.5 in

111 both conditions. These data clearly demonstrate that in the absence of alpha-

112 synuclein, the dynamics population number in SN neurons is changed.

113 Additionally, TH-positive cells were counted in VTA on E13.5 day when midbrain

114 dopaminergic neurons are known to separate into SN and VTA zones (Hu et al.

115 2004). The positioning of SN and VTA in embryonic mouse brain for stereological

116 counting of DA neutrons is shown on Fig 2. As a result, we found that embryonic

117 DA neurons in VTA are not affected in alpha-synuclein knockout mice and their

118 number is the same as in WT control embryonic brains (Fig.1B).

119

120

\section{Discussion}

121 The role of alpha-synuclein in the genesis of dopaminergic neurons of the SN has

122 been investigated in several previous studies (Garcia-Reitboeck et al. 2013;

123 Spillantini \& Goedert 2017; Zaltieri et al. 2015). Loss of function of alpha-synuclein

124 leads to a reduced number of DA neurons in SN at E13.5 (Garcia-Reitboeck et al.

125 2013) and this deficit remains in adulthood (Robertson et al. 2004). Here using

126 littermates as mothers for producing embryos at consecutive stages of embryonic

127 development, we have conducted a precise study of DA neurons in the SN, and have

128 shown that not only the number of E13.5 DA neurons is reduced, but that the way

129 these neurons are formed is changed in alpha-synuclein knockout mice (Fig.1A).

130 Therefore, alpha-synuclein deficit leads to an inefficient DA neuron pool formation

131 in SN during embryonic development.

132 It is well established that the degeneration of DA neurons of VTA is less prominent

133 in PD brains (Alberico et al. 2015; Mosharov et al. 2009) and that overexpression of

134 the PD mutant alpha-synuclein does not affect VTA (Maingay et al. 2006). In our

135 study no changes were found in number of DA neurons of VTA. These confirm that

136 alpha-synuclein among other factors responsible for the differential sensitivity of SN

137 DA neurons to damage in PD pathology. Further investigation is warranted to

138 determine whether alpha-synuclein deficiency can shape the differential

139 vulnerability of DA neurons to neurodegeneration in the adult and aged brain. 


\section{Conclusion}

142 Our data have shown a prominent modulating effect of alpha-synuclein on the 143 formation of DA neurons of the SN and no effect on the formation of DA neurons 144 in VTA suggesting a critical role for this protein in maturation of the population of 145 DA neurons in substantia nigra.

147 Acknowledgments

148 Part of the experimental work was performed using the equipment of Center for 149 collective use of Institute of Physiologically Active Compounds Russian Academy 150 of Sciences. 


\section{References}

152

153

154

155

156

157

158

159

160

161

162

163

164

165

166

167

168

169

170

171

172

173

174

175

176

177

178

179

180

181

182

183

184

185

186

187

188

189

190

191

192

193
Abeliovich A, and Hammond R. 2007. Midbrain dopamine neuron differentiation: factors and fates. Dev Biol 304:447-454. 10.1016/j.ydbio.2007.01.032

Abeliovich A, Schmitz Y, Farinas I, Choi-Lundberg D, Ho WH, Castillo PE, Shinsky N, Verdugo JM, Armanini M, Ryan A, Hynes M, Phillips H, Sulzer D, and Rosenthal A. 2000. Mice lacking alpha-synuclein display functional deficits in the nigrostriatal dopamine system. Neuron 25:239-252.

Al-Wandi A, Ninkina N, Millership S, Williamson SJ, Jones PA, and Buchman VL. 2010. Absence of alpha-synuclein affects dopamine metabolism and synaptic markers in the striatum of aging mice. Neurobiol Aging 31:796-804. 10.1016/j.neurobiolaging.2008.11.001

Alberico SL, Cassell MD, and Narayanan NS. 2015. The Vulnerable Ventral Tegmental Area in Parkinson's Disease. Basal Ganglia 5:51-55. 10.1016/j.baga.2015.06.001

Burre J, Sharma M, Tsetsenis T, Buchman V, Etherton MR, and Sudhof TC. 2010. Alphasynuclein promotes SNARE-complex assembly in vivo and in vitro. Science 329:1663-1667. 10.1126/science.1195227

Garcia-Reitboeck P, Anichtchik O, Dalley JW, Ninkina N, Tofaris GK, Buchman VL, and Spillantini MG. 2013. Endogenous alpha-synuclein influences the number of dopaminergic neurons in mouse substantia nigra. Exp Neurol 248:541-545. 10.1016/j.expneurol.2013.07.015

Goedert M, Jakes R, and Spillantini MG. 2017. The Synucleinopathies: Twenty Years On.J Parkinsons Dis 7:S53-S71. 10.3233/JPD-179005

Gorbatyuk OS, Li S, Nash K, Gorbatyuk M, Lewin AS, Sullivan LF, Mandel RJ, Chen W, Meyers C, Manfredsson FP, and Muzyczka N. 2010. In vivo RNAi-mediated alpha-synuclein silencing induces nigrostriatal degeneration. Mol Ther 18:1450-1457. $10.1038 / \mathrm{mt} .2010 .115$

Greten-Harrison B, Polydoro M, Morimoto-Tomita M, Diao L, Williams AM, Nie EH, Makani S, Tian N, Castillo PE, Buchman VL, and Chandra SS. 2010. alphabetagammaSynuclein triple knockout mice reveal age-dependent neuronal dysfunction. Proc Natl Acad Sci U S A 107:19573-19578. 10.1073/pnas.1005005107

Hu Z, Cooper M, Crockett DP, and Zhou R. 2004. Differentiation of the midbrain dopaminergic pathways during mouse development. J Comp Neurol 476:301-311. $10.1002 /$ cne.20230

Janezic S, Threlfell S, Dodson PD, Dowie MJ, Taylor TN, Potgieter D, Parkkinen L, Senior SL, Anwar S, Ryan B, Deltheil T, Kosillo P, Cioroch M, Wagner K, Ansorge O, Bannerman DM, Bolam JP, Magill PJ, Cragg SJ, and Wade-Martins R. 2013. Deficits in dopaminergic transmission precede neuron loss and dysfunction in a new Parkinson model. Proc Natl Acad Sci U S A 110:E4016-4025. $10.1073 /$ pnas. 1309143110

Ludtmann MH, Angelova PR, Ninkina NN, Gandhi S, Buchman VL, and Abramov AY. 2016. Monomeric Alpha-Synuclein Exerts a Physiological Role on Brain ATP Synthase.J Neurosci 36:10510-10521. 10.1523/JNEUROSCI.1659-16.2016 
194 Luo SX, and Huang EJ. 2016. Dopaminergic Neurons and Brain Reward Pathways: From

195

196

197

198

199

200

201

202

203

204

205

206

207

208

209

210

211

212

213

214

215

216

217

218

219

220

221

222

223

224

225

226

227

228

229
Neurogenesis to Circuit Assembly. Am J Pathol 186:478-488. 10.1016/j.ajpath.2015.09.023

Maingay M, Romero-Ramos M, Carta M, and Kirik D. 2006. Ventral tegmental area dopamine neurons are resistant to human mutant alpha-synuclein overexpression. Neurobiol Dis 23:522-532. 10.1016/j.nbd.2006.04.007

Mosharov EV, Larsen KE, Kanter E, Phillips KA, Wilson K, Schmitz Y, Krantz DE, Kobayashi K, Edwards RH, and Sulzer D. 2009. Interplay between cytosolic dopamine, calcium, and alpha-synuclein causes selective death of substantia nigra neurons. Neuron 62:218-229. 10.1016/j.neuron.2009.01.033

Nemani VM, Lu W, Berge V, Nakamura K, Onoa B, Lee MK, Chaudhry FA, Nicoll RA, and Edwards RH. 2010. Increased expression of alpha-synuclein reduces neurotransmitter release by inhibiting synaptic vesicle reclustering after endocytosis. Neuron 65:66-79. 10.1016/j.neuron.2009.12.023

Ninkina N, Peters OM, Connor-Robson N, Lytkina O, Sharfeddin E, and Buchman VL. 2012. Contrasting effects of alpha-synuclein and gamma-synuclein on the phenotype of cysteine string protein alpha (CSPalpha) null mutant mice suggest distinct function of these proteins in neuronal synapses. J Biol Chem 287:44471-44477. $10.1074 /$ jbc.M112.422402

Robertson DC, Schmidt O, Ninkina N, Jones PA, Sharkey J, and Buchman VL. 2004. Developmental loss and resistance to MPTP toxicity of dopaminergic neurones in substantia nigra pars compacta of gamma-synuclein, alpha-synuclein and double alpha/gamma-synuclein null mutant mice. J Neurochem 89:1126-1136. 10.1111/j.1471-4159.2004.02378.x

Ryan BJ, Hoek S, Fon EA, and Wade-Martins R. 2015. Mitochondrial dysfunction and mitophagy in Parkinson's: from familial to sporadic disease. Trends Biochem Sci 40:200-210. 10.1016/j.tibs.2015.02.003

Spillantini MG, and Goedert M. 2017. Neurodegeneration and the ordered assembly of alpha-synuclein. Cell Tissue Res. 10.1007/s00441-017-2706-9

Vargas KJ, Schrod N, Davis T, Fernandez-Busnadiego R, Taguchi YV, Laugks U, Lucic V, and Chandra SS. 2017. Synucleins Have Multiple Effects on Presynaptic Architecture. Cell Rep 18:161-173. 10.1016/j.celrep.2016.12.023

Zaltieri M, Grigoletto J, Longhena F, Navarria L, Favero G, Castrezzati S, Colivicchi MA, Della Corte L, Rezzani R, Pizzi M, Benfenati F, Spillantini MG, Missale C, Spano P, and Bellucci A. 2015. alpha-synuclein and synapsin III cooperatively regulate synaptic function in dopamine neurons. J Cell Sci 128:2231-2243. 10.1242/jcs.157867 


\section{Figure 1 (on next page)}

The number of TH-positive neurons in SN of alpha-synuclein knockout and control WT mice in embryogenesis (A); the number of TH-positive neurons in VTA, E13.5 (B).

Values represent means \pm S.E.M. $n=5$ for each group and time points. Two-way ANOVA, $(* * * p<0.001, * p<0.05)$. 

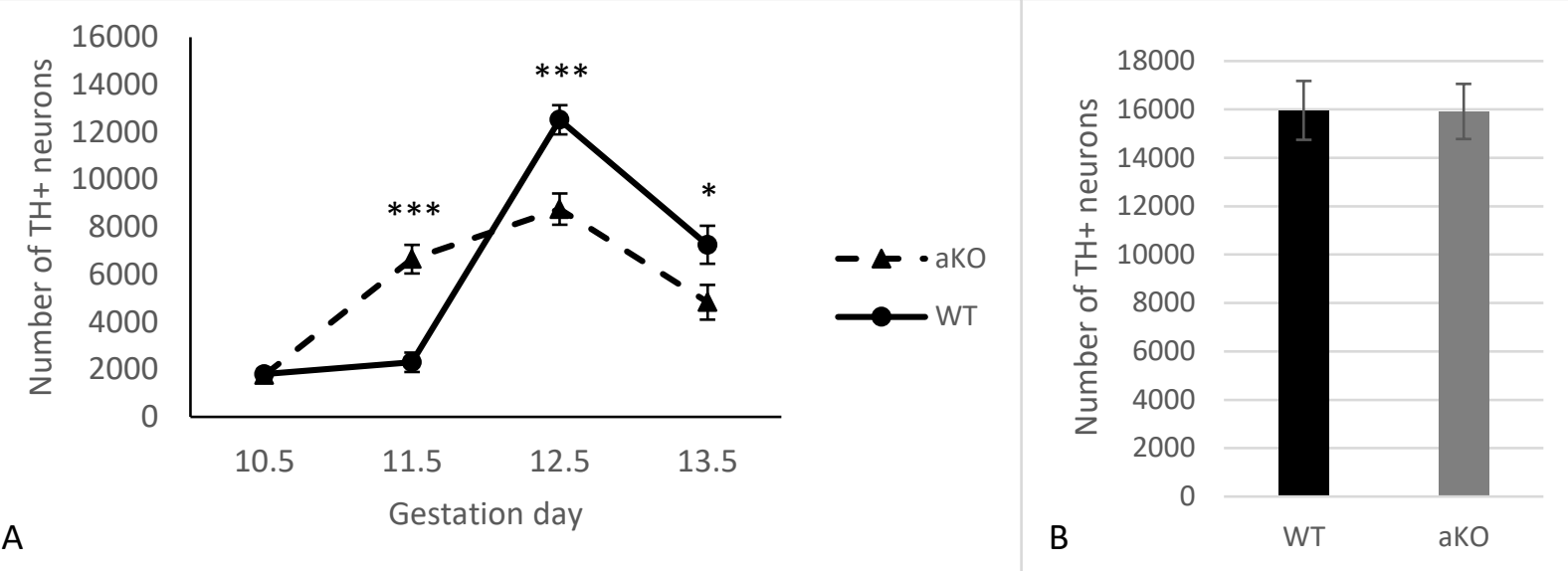


\section{Figure 2}

A representative developing brain sections from WT $(A, C)$ and aKO $(B, D)$ mice on 11.5 $(A, B)$ and $13.5(C, D)$ embryonic days immunostained with anti-tyrosine hydroxylase $(T H)$ antibody.

The areas corresponding to the Substantia Nigra (SN) and Ventral Tegmental Area (VTA) are outlined by the line. TH-positive neurons indicated by arrows at a higher magnification (inserts). 

